\title{
A TWO-POINT SET MUST BE ZERO-DIMENSIONAL
}

\author{
JOHN KULESZA
}

(Communicated by Andreas R. Blass)

\begin{abstract}
We prove that any subset of $\mathbb{R}^{2}$ that intersects no line in more than two points either is zero-dimensional or contains an arc. This is used to prove that any subset of $\mathbb{R}^{2}$ that intersects each line in exactly two points is zero-dimensional, answering a question of Mauldin.
\end{abstract}

A subset of $\mathbb{R}^{2}$ is called a partial two-point set if it meets each line in at most two points and is called a two-point set if it meets each line in exactly two points. The existence of a two-point set, using the axiom of choice, was shown by Mazurkiewicz in [Ma]. In [M] Mauldin lists three open questions involving two-point sets. Our Theorem 2 provides a positive answer to the second of these questions, "Must every two-point set be zero-dimensional?". Mauldin has shown, as mentioned in [M], that a two-point set must always be totally disconnected (meaning the only connected subsets are points); however, there are many examples of totally disconnected subsets of $\mathbb{R}^{2}$ that are not zero-dimensional.

There are partial two-point sets that are not zero-dimensional; a circle is such a set. Theorem 1 states that a partial two-point set, in order to be positivedimensional, must contain an arc. Taken together with Mauldin's result, this answers the question. However, since Mauldin's result is unpublished, we give an alternate solution, also using Theorem 1 .

We remark that the third question in [M] is misstated and, as stated, has a negative answer. A circle with a countable dense subset removed demonstrates this. The correct version is "Can all compact zero-dimensional partial two-point sets be extended to two-point sets?".

We also remark that the first question in [M], "Can a two-point set be Borel?", the main question involving two-point sets, remains unanswered.

\section{Preliminaries}

If $X$ is a topological space and $x \in X$, then $X$ is said to be zero-dimensional at $x$, denoted $\operatorname{ind}(X, x)=0$, if whenever $O$ is an open set containing $x$ there is a simultaneously open and closed $O^{\prime}$ such that $x \in O^{\prime} \subseteq O$, and $X$ is said to be zero-dimensional, denoted $\operatorname{ind}(X)=0$, if it is zero-dimensional at each

Received by the editors January 22, 1991 and, in revised form, March 4, 1991.

1991 Mathematics Subject Classification. Primary 54B05, 54H05, 54F45.

Key words and phrases. Zero-dimensional, two-point set. 
$x \in X$. The ind notation refers to the small inductive dimension, but for separable metric spaces, all of the usual notions of dimension coincide. For a complete discussion of dimension theory, the reader is referred to [E].

For our purposes, arcs are sets homeomorphic to $[0,1]$, while lines, line segments, and rays are assumed to be "straight".

If $p$ and $q$ are points, then $l_{p q}$ denotes the line determined by $p$ and $q$; if $l$ is a line, $C(l)^{0}$ and $C(l)^{1}$ denote the components of the complement of $l$, chosen arbitrarily. By $\operatorname{Box}(p, q, \varepsilon, \delta)$ we mean the open rectangular region with two sides formed by line segments of the two lines perpendicular to $l_{p q}$ that intersect $l_{p q}$ at a distance of $\varepsilon$ from $p$ and the other two sides formed by line segments of the two lines parallel to $l_{p q}$ that are at a distance $\delta$ from $l_{p q}$. For each pair of points $p$ and $q, B_{p q}=\{\operatorname{Box}(p, q, \varepsilon, \delta): \varepsilon, \delta>0\}$ forms a local base at $p$. If $X$ is a partial two-point set and $p \in X$, then for any $q$, $B_{p q}^{*}=\left\{B \in B_{p q}\right.$ : the sides of $B$ perpendicular to $l_{p q}$ do not intersect $\left.X\right\}$ also forms a local base at $p$.

\section{Results}

Our goal is to first prove Theorem 1, a general fact about partial two point sets, and then use it to prove Theorem 2, which answers Mauldin's question.

Theorem 1. If $X$ is a partial two-point set then ind $(X)>0$ if and only if $X$ contains an arc.

Before proving Theorem 1, we prove two lemmas.

Lemma 1. Suppose $X$ is a partial two-point set, $p$ and $q$ are distinct members of $\mathbb{R}^{2}, p \in \operatorname{cl}\left(X \cap C\left(l_{p q}\right)^{i}\right)$ for each $i \in 2$ and $q \in \operatorname{cl}(X)$. If $x \in\left(l_{p q} \cap X\right) \backslash\{p, q\}$ then $\operatorname{ind}(X, x)=0$.

Proof. Assume $0<\varepsilon<\min \{d(x, p), d(x, q)\}$, and $B=\operatorname{Box}(x, p, \varepsilon, \delta) \in$ $B_{x p}^{*}$. The boundary of $B$ in $X$ is contained in the two sides parallel to $l_{p q}$. We show how to replace these sides with new sides that have no boundary in $X$. For simplicity, we assume that $q \in X$; if not, the argument can be adjusted using a point of $X$ very close to $q$. Then, for $i \in 2$, we can find $p_{i} \in C\left(l_{p q}\right)^{i} \cap X$ close enough to $p$ so that the line $l_{p_{i}}$ intersects both sides of $B$ that are perpendicular to $l_{p q}$. The segments determined by these intersections do no intersect $X$ since both $p_{i}$ and $q$ are in $X$. Then the open, bounded region determined by the lines $l_{p_{0} q}, l_{p_{1} q}$, and the sides of $B$ perpendicular to $l_{p q}$ has no boundary in $X$, contains $x$, and is contained in $B$. Hence $\operatorname{ind}(X, x)=0$.

Lemma 2. If $X$ is a partial two-point set and $\operatorname{ind}(X)>0$, then $\operatorname{cl}(X)$ contains no line segment.

Proof. Suppose $I$ is a line segment in $\operatorname{cl}(X)$ with end points $a$ and $b$ and $x \in X$ not on the line determined by $I$. There is a point $q \in X$ such that $l_{x q} \cap I=\{p\}$, where $p \notin\{a, b\}$. Applying Lemma 1 , ind $(X, x)=0$. Since there are only, at most, two points of $X$ on the line determined by $I$ and finitely many points do not alter the dimension of a set, $\operatorname{ind}(X)=0$.

Proof of Theorem 1. One direction is obvious. For the other, we assume ind $(X)$ $>0$ and also that $X$ contains no arc. Fix $x \in X$ such that $\operatorname{ind}(X, x)>0$. We aim for a contradiction. 
By translating $X$, we may assume that $x$ is the origin; we consider the unit circle $S$ as parametrized by the interval $[0,2 \pi)$ in the obvious fashion. For each $\alpha \in S$, let $r_{\alpha}$ denote the ray emanating from $x$ and passing through $\alpha$, and let $I_{\alpha}$ denote the line containing $r_{\alpha}$. Let $B$ be a basis of open arcs on $S$ whose closures do not contain all of $S$. For $K \subseteq S$, let $\operatorname{sect}(K)=$ $\left(\bigcup\left\{r_{\alpha}: \alpha \in K\right\}\right) \backslash\{x\}$.

Suppose $\mathscr{O} \in B$, with endpoints $\delta$ and $\varepsilon$, such that $\operatorname{sect}(\operatorname{cl}(\mathscr{O}) \cap \operatorname{cl}(X))$ contains a continuum $C$, missing $x$, from $r_{\delta}$ to $r_{\varepsilon}$. We claim that $\operatorname{ind}(X \cap \operatorname{sect}(\mathscr{O}))=0$. For each $\alpha \in \mathscr{O}$ there is a point $p_{\alpha} \in C \cap r_{\alpha}$ with $p$ $\in \operatorname{cl}\left(X \cap C\left(l_{\alpha}\right)^{i}\right)$ for each $i \in 2$. If, for some $\alpha \in 0$, there were a point $q_{\alpha} \in$ $\operatorname{cl}(X) \cap r_{\alpha}$ and distinct from $p_{\alpha}$, then Lemma 1 would imply that $\operatorname{ind}(X, x)=$ 0 . Thus $\operatorname{cl}(X) \cap r_{\alpha}=\left\{p_{\alpha}\right\}$. This implies that $\operatorname{cl}(X) \cap \operatorname{sect}(\mathscr{O})$, is homeomorphic to $\mathbb{R}$, since the radial projection of $\operatorname{cl}(X) \cap \operatorname{sect}(\mathscr{O})$ to the unit circle is a homeomorphism. Since $X$ contains no arc, ind $(X \cap \operatorname{sect}(\mathscr{O}))=0$.

Let $B^{\prime}=\{\mathscr{O} \in B: \operatorname{ind}(\operatorname{sect}(\mathscr{O}) \cap X)=0\}$ and let $Y=\operatorname{sect}\left(\bigcup B^{\prime}\right)$. Then ind $(Y)=0$ since it is a union of countably many zero-dimensional closed subsets of $X$. Let $Z=X \backslash Y . Z$ is a closed subset of $X$. If ind $(Z) \neq 0$ then $\operatorname{cl}(Z)$ contains a continuum, which by the choice of $B^{\prime}$ must contain a line segment on a radial line from $x$. But this is not possible by Lemma 2 . So $X$ is the union of a zero-dimensional subset and a closed zero-dimensional subset; hence $\operatorname{ind}(X)=0$. This is a contradiction.

The next lemma allows us to answer Mauldin's question.

Lemma 3. A two-point set cannot contain an arc.

Proof. Let $X$ be a two-point set, let $A$ be an arc, and suppose $A \subseteq X$. Let $p$ and $q$ be the end points of $A$, and $l$ be the line through $p$ and $q$. Then $A$ intersects only one component of the complement of $l$. Let $m$ be the line parallel to $l$ in that component that intersects $A$ and is the farthest such line from $l$ to do so. Then either $|A \cap m|=1$ or $|A \cap m|=2$. If $|A \cap m|=2$, then for lines parallel to $m$, between $l$ and $m$, and close enough to $m$, there will be at least four points of intersection with $A$. If $|A \cap m|=1$, then let $x$ denote the point of $m \cap X \backslash A$. Either $\left|l_{p x} \cap A\right| \geq 2$ or $\left|l_{q x} \cap A\right| \geq 2$, say $\left|l_{p x} \cap A\right| \geq 2$. But then $\left|l_{p x} \cap X\right|=\left|\left(l_{p x} \cap A\right) \cup\{x\}\right| \geq 3$. Hence we conclude $X$ is not a two point set.

Theorem 1 and Lemma 3 taken together give

Theorem 2. If $X$ is a two-point set then $X$ is zero-dimensional.

\section{REFERENCES}

[E] R. Engelking, Dimension theory, PWN, Warszawa, 1978.

[M] R. D. Mauldin, Problems in topology, Open Problems in Topology (J. van Mill and G. M. Reed, eds.), North-Holland, Amsterdam, 1990.

[M] S. Mazurkiewicz, Sur un ensemble plan qui a avec chaque droite deux et seulement deux points communs, C. R. Varsovie 7 (1914), 382-384.

Department of Mathematics, George Mason University, Fairfax, Virginia 22030 